Draft VERSion SEPTEMBer 9, 2021

Preprint typeset using LATEX $_{\mathrm{E}} \mathrm{X}$ style emulateapj v. 08/22/09

\title{
REGULATION OF THERMAL CONDUCTIVITY IN HOT GALAXY CLUSTERS BY MHD TURBULENCE
}

\author{
Steven A. Balbus ${ }^{1}$ and Christopher S. Reynolds ${ }^{2}$ \\ Draft version September 9, 2021
}

\begin{abstract}
The role of thermal conduction in regulating the thermal behavior of cooling flows in galaxy clusters is reexamined. Recent investigations have shown that the anisotropic Coulomb heat flux caused by a magnetic field in a dilute plasma drives a dynamical instability. A long standing problem of cooling flow theory has been to understand how thermal conduction can offset radiative core losses without completely preventing them. In this Letter, we propose that magnetohydrodynamic turbulence driven by the heat flux instability regulates field-line insulation and drives a reverse convective thermal flux, both of which may mediate the stabilization of the cooling cores of hot clusters. This model suggests that turbulent mixing should accompany strong thermal gradients in cooling flows. This prediction seems to be supported by the spatial distribution of metals in the central galaxies of clusters, which shows a much stronger correlation with the ambient hot gas temperature gradient than with the parent stellar population.
\end{abstract}

Subject headings: cooling flows - galaxies: clusters: general - magnetic fields - MHD — instabilities - turbulence

\section{INTRODUCTION}

Although the intracluster medium (ICM) of galaxy clusters has been an active area of study since the earliest days of X-ray astronomy (Felten 1966), the complexity of the physical processes regulating the behavior of this gas continues to surprise us. X-ray observations imply that cooling times in the core regions of ICM atmospheres are significantly shorter than cluster ages (Peterson \& Fabian 2006). Prior to the launch of XMM-Newton and Chandra, it was widely held that these short cooling times lead to flows in which mass is dropping out (Fabian 1994). Subsequent XMM-Newton spectroscopy confirmed the short cooling times and inwardly decreasing ICM temperature profiles, but also revealed a lack of gas cooling below temperatures of $T \sim T_{\mathrm{vir}} / 3$, where $T_{\mathrm{vir}}$ is the virial temperature of the cluster (Peterson et al. 2001; Tamura et al. 2001). This observation, combined with a realization that unchecked cooling flows would lead to central galaxies far above the high-end cutoff of the luminosity function (Benson et al. 2003), made it clear that the cooling cores of galaxy clusters must be continuously heated.

A new paradigm consisting of core heating by a central radio galaxy has taken hold. Although a few examples were known at the time of ROSAT, Chandra and $X M M-N e w t o n$ have observed cavities and weak shocks in the ICM of many clusters that are driven by the central radio-galaxy (e.g., Fabian et al. 2000, 2003; Young et al. 2002; Heinz et al. 2002). This has motivated the hypothesis that core losses are offset by thermalization of the kinetic luminosity of the jets from the active galactic nucleus (AGN). In some (but not all; see §4) systems, the energetics of the AGN is comparable to the radiative luminosity of the cluster core (Birzan et al. 2004; Best et al. 2007). AGN feedback of this form has been adopted

\footnotetext{
${ }^{1}$ Laboratoire de Radioastronomie, École Normale Superiéure, 24 rue Lhomond, 75231 Paris CEDEX 05

2 Department of Astronomy, University of Maryland, College Park, MD 20742-2421
}

by semi-analytic galaxy formation theories in the guise of radio-mode feedback (Croton et al. 2006).

An alternative hypothesis is that thermal conduction into the core from the large heat reservoir beyond the cooling radius may offset the radiative loss (Binney \& Cowie 1981). A source of uncertainty for such models is the factor $f$ by which magnetic fields suppress thermal conduction below the classical Spitzer value $\kappa_{S}$ (Spitzer 1962). Plausible estimates range from $f \sim 10^{-2}$ (Churazov et al. 2001) to $f \sim 0.3$ (Narayan \& Medvedev 2001). Thermal conduction models have faced other significant challenges, however. Even unbridled Spitzer conductivity cannot offset radiative cooling in the lowermass/cooler clusters (Voigt and Fabian 2004), a consequence of the strong temperature dependence of Spitzer conductivity, $\kappa_{S} \propto T^{5 / 2}$. For hotter clusters, thermal conduction can in principle offset radiative cooling if $f \sim 0.1-1$ (Zakamska \& Narayan 2003). In this case, however, fine tuning of $f$ is required to offset radiative cooling, yet not erase the observed temperature gradients within the cooling core (e.g., see Conroy \& Ostriker 2008). Furthermore, assuming $f$ is a constant for a given ICM atmosphere, these equilibria are still thermally unstable (Kim \& Narayan 2003).

In this Letter, we argue that recent theoretical developments in the theory of the stability of MHD atmospheres must have a crucial bearing on the behavior of cluster cooling cores. In particular, it is now understood that in atmospheres of dilute plasmas (such as the ICM) in which thermal conduction is restricted to follow magnetic field lines, the convective instability criterion is fundamentally altered: temperature gradients, not entropy gradients, are crucial. Atmospheres in which the temperature gradient is decreasing upwards $(d T / d z<0)$, but field lines are isothermal, are convectively unstable (Balbus 2000, 2001; Parrish \& Stone 2005, 2007) even when the entropy gradient would normally imply stability. On the other hand, atmospheres in which the temperature gradient is increasing upwards $(d T / d z>0$; 
the case in cooling cores), and field lines are thermally conducting, are also unstable (Quataert 2008; Parrish \& Quataert 2008). These two powerful instabilities are known respectively as the magnetothermal instability (MTI; $d T / d z<0$ ) and the heat flux-driven buoyancy instability ( $\mathrm{HBI} ; d T / d z>0$ ).

Our focus is the cooling core of a rich/hot cluster of galaxies. We investigate the consequences of convective turbulence that is driven, but also regulated, by the HBI. Turbulence, far from being a source of heat, is actually a cooling term in the energy balance. The role of convective fluctuations is an outwardly-directed "return heat flux" that, together with field line insulation, lowers, but by no means erases, the inward diffusive thermal flux. This process eliminates the theoretical ad hoc fine tuning required of previous conduction-based models. Since our picture attributes the stabilization of hot cooling cores to the intrinsic dynamics of MHD plasmas, the observed homology of hot cooling cores range is perhaps not surprising. Moreover, turbulence-regulated heat conduction also accounts for the otherwise puzzling tight correlation between metalicity distribution and temperature gradient observed in cluster cores: the two become intimately related to the presence of convective turbulence.

An outline of this Letter is as follows. In $\S 2$, we discuss the role of radiative losses on the HBI in cluster cooling flows. The role of convective heat transport is studied in $\S 3$, and our picture for cooling cores is explicitly discussed in $\S 4$. We summarize our conclusions in $\S 5$.

\section{RADIATIVE COOLING EFFECTS ON THE HBI}

Before presenting our thermal model of hot cluster cooling cores, it is of interest to modify the HBI dispersion relation of Quataert (2008) due to the presence of radiative losses. We restrict our calculation to the simplest and most illustrative case of a uniform background magnetic field in the vertical $z$ direction.

Radiative losses are represented by the net cooling function $-\rho \mathcal{L}$ (units erg s $\mathrm{s}^{-1} \mathrm{~cm}^{-3}$ ); this terms includes any explicit heating processes as well as radiative cooling. We make the formal assumption that the background is static. To include first-order perturbations in radiative loses, the term $-\delta(\rho \mathcal{L})$ should be added to the right side of equation (11) in Q08. Plane wave disturbances of the form $\exp \left(\sigma t+i k_{x} x+i k_{z} z\right)$ then satisfy the dispersion relation

$$
\sigma_{\text {therm }} \sigma_{d y n}^{2}+\frac{k_{x}^{2}}{k^{2}} \sigma N^{2}-\frac{2}{5} \kappa \frac{k_{z}^{2} k_{x}^{2}}{k^{2}} g \frac{d \ln T}{d z}=0
$$

where

$$
\begin{gathered}
\sigma_{\text {therm }}=\sigma+\frac{2}{5} \kappa k_{z}^{2}+\left[\frac{2 T}{5 P}\left(\frac{\partial(\rho \mathcal{L})}{\partial T}\right)_{P}\right] \\
\sigma_{d y n}^{2}=\sigma^{2}+\left(\boldsymbol{k} \cdot \boldsymbol{v}_{\boldsymbol{A}}\right)^{2}
\end{gathered}
$$

We have written $\sigma$ for $-i \omega$ in Q08, otherwise the notation is identical. (The quantity $g$ is $-(1 / \rho) d P / d z$.)

For the case of a vertical field, equation (11) corresponds precisely to the dispersion relation (13) of Q08, but with the additional cooling term in square brackets in equation
(2). The constant term in the dispersion relation (11) is

$$
\left(\boldsymbol{k} \cdot \boldsymbol{v}_{\boldsymbol{A}}\right)^{2}\left[\frac{2}{5} \kappa k_{z}^{2}+\frac{2 T}{5 P}\left(\frac{\partial(\rho \mathcal{L})}{\partial T}\right)_{P}\right]-\frac{2}{5} \kappa \frac{k_{z}^{2} k_{x}^{2}}{k^{2}} g \frac{d \ln T}{d z}
$$

This should be negative for instability. In the limit of very small $\boldsymbol{k} \cdot \boldsymbol{v}_{\boldsymbol{A}}$, we recover the HBI condition that an increasing temperature profile is unstable. We see, however, that in the presence of a non-negligible magnetic field, radiative cooling (which, in this setting, has $\left.(\partial(\rho \mathcal{L}) / \partial T)_{P}<0\right)$ acts to further destabilize the atmosphere, especially at longer wavelengths.

The non-linear behavior of an HBI unstable atmosphere is still unclear. On the basis of 2D and 3D simulations of non-cooling plane-parallel atmospheres, Parrish \& Quataert (2008; hereafter PQ08) show that the HBI acts to re-orient field lines perpendicular to the temperature gradient, effectively shutting off the conductive heat flux. We shall refer to this process as field-line insulation. However, it is not obvious that the conclusions of PQ08 can be extended to actual cluster cooling cores. As well as the topological impossibility of establishing a (nonzero) field configuration that has zero radial field everywhere, the inflow of matter associated with unbalanced radiative cooling in an insulated core will readily generate a radial field from existing transverse field. Thus, we argue that field-line insulation, while surely present at some level, cannot be absolute in cluster cooling cores.

One of the most salient properties of the linear HBI is its convective nature, in particular the fact that the product $\delta v_{z} \delta T>0$ (Q08). If sufficiently large, this reverse flux regulates the background heat flux: the greater the magnitude of $d T / d z$, the more vigorous the return flux. We suggest that the combination of field line insulation and reverse convective flux fixes a state of marginal stability to the radiative HBI that corresponds to the cool cores of galaxy clusters.

\section{HEAT TRANSPORT WITHIN HOT CLUSTER COOLING CORES}

Let us now re-consider the energy equation for the cooling core of a hot cluster in the light of the above discussion. Under steady conditions, the equation of hydrodynamical energy conservation is

$$
\boldsymbol{\nabla} \cdot\left[\left(\frac{v^{2}}{2}+\frac{5 P}{2 \rho}+\Phi\right) \rho \boldsymbol{v}+\boldsymbol{Q}\right]=-\rho \mathcal{L},
$$

where $\Phi$ is the potential function of the cluster, and $\boldsymbol{Q}=$ $\kappa_{S} \boldsymbol{b}(\boldsymbol{b} \cdot \boldsymbol{\nabla} T)$ is the diffusive heat flux ( $\boldsymbol{b}$ is a unit vector in the direction of the magnetic field).

We wish to investigate the nature of dynamically static solutions to this equation in which, on average, the mass flux $\rho \boldsymbol{v}$ vanishes. The HBI is assumed to be present and fully developed into convective turbulence. This requires a background heat flux $\boldsymbol{Q}$ to be present, since this is the seat of the instability. We assume that the density and temperature consist of a mean background value plus a fluctuation, $\rho=\rho_{0}+\delta \rho, \quad T=T_{0}+\delta T$. The radial velocity $v$ (the only component of concern here) is dominated by its fluctuating component, but must also have a very small second order part with a finite mean value, $v_{2}$. This is needed if the mass flux vanishes,

$$
\left\langle\left(\rho_{0}+\delta \rho\right)\left(v_{2}+\delta v\right)\right\rangle \simeq \rho_{0} v_{2}+\langle\delta \rho \delta v\rangle=0
$$


where \langle\rangle indicates a time average. Defining the normalized temperature $\tau=P / \rho$, our energy equation reads

$$
\boldsymbol{\nabla} \cdot\left[\frac{5}{2} \rho_{o}\langle\delta \boldsymbol{v} \delta \tau\rangle+\boldsymbol{Q}\right]=-\rho \mathcal{L}
$$

This explicitly demonstrates that it is the divergence of both the (inwardly directed) diffusive heat flux $\boldsymbol{Q}$ and the (outwardly-directed) turbulent heat flux $(5 / 2) \rho\langle\delta \boldsymbol{v} \delta \tau\rangle$ that, in our model, balances radiative cooling. Notice that, as in Schwarzschild (1958), the convective heat flux is proportional to the correlation of velocity and temperature fluctuations.

We now estimate the outwardly directed convective heat flux $(5 / 2) \rho\langle\delta \boldsymbol{v} \delta \tau\rangle$, using arguments similar to Schwarzschild (1958). Henceforth, we drop the 0 subscript: $\rho$ and $T$ are understood to be background values. The fastest growing modes of the HBI typically have (Q08)

$$
\frac{\delta \tau}{\tau}=\frac{\delta T}{T}=\xi \frac{d \ln T}{d r}
$$

and density deficits

$$
|\delta \rho|=\rho \xi \frac{d \ln T}{d r},
$$

where $\xi$ is the radial displacement. Following Schwarzschild, we multiply this density deficit by $g \xi / 2$ to obtain the kinetic energy per unit volume of a rising element, $\rho(\delta v)^{2} / 2$ :

$$
\frac{\rho(\delta v)^{2}}{2}=\frac{\rho g}{2} \xi^{2}\left(\frac{d \ln T}{d r}\right)
$$

or

$$
\delta v=\xi g^{1 / 2}\left(\frac{d \ln T}{d r}\right)^{1 / 2}
$$

Therefore, the convective heat flux is

$$
\frac{5}{2} \rho\langle\delta v \delta \tau\rangle=\frac{5}{2} P g^{1 / 2}\left(\frac{d \ln T}{d r}\right)^{3 / 2} \xi^{2}=\frac{5}{2} P\left(\frac{d \ln T}{d r}\right) \mathcal{D},
$$

where $\mathcal{D}$ is the turbulent diffusion coefficient

$$
\mathcal{D} \equiv\langle\xi \delta v\rangle=\xi^{2} g^{1 / 2}\left(\frac{d \ln T}{d r}\right)^{1 / 2} .
$$

Schwarzschild identifies $\xi$ with $l / 2$, where $l$ is a formal mixing length, but such details are of secondary concern here. The main point is that if these basic scalings are correct, the outward convective heat flux acts like a reverse diffusive heat flux, but one in which the thermal conductivity is itself proportional to $(d T / d r)^{1 / 2}$. Together with incomplete field-line insulation, this is wellsuited to act as an efficient regulatory mechanism counteracting the otherwise large inward diffusive transport of thermal energy.

\section{DISCUSSION}

HBI-driven turbulence has two important consequences for heat transport in hot cluster cores, the partial suppression of the conductive heat flux due to fieldline re-orientation (previously stressed by PQ08) and an outwardly-directed convective heat flux (highlighted in this work). We hypothesize here that these two effects keep a cooling core in marginal thermal stability, with the (regulated) thermal conduction balancing radiative cooling.

Consider a hot cluster with an initially isothermal ICM, lacking any embedded heat source such as an AGN. Cooling in the core of the cluster would set up an initially small temperature gradient and, for a short time, the radiative loses would be easily compensated by thermal conduction down this temperature gradient. As time proceeds, however, HBI induced field-line insulation and the convective heat flux will act to suppress the conduction heat flux, leading to a steepening temperature gradient. As the center of the cluster approaches a cooling catastrophe, the vigorous turbulence will comb the field lines of the turbulent ICM preferentially in the radial direction, opening up an enhanced channel for the inwards conductive heat flux and quenching the runaway cooling. We posit that dense ICM cores will evolve towards a state of near static, marginal "radiative-HBI" stability, and identify this with the observed state of the hot cluster cores.

Although this work appears to be the first to invoke turbulence as a cooling mechanism, the suggestion that cluster cooling cores may be turbulent is certainly not new (e.g., see Voigt \& Fabian 2004). Indeed, the presence of turbulence is motivated by observational studies of the rotation measure towards embedded radio sources (e.g., Vogt \& Ensslin 2005), spatially resolved ICM pressure fluctuations (Schuecker et al. 2004), and X-ray resonant line scattering (e.g., Gastaldello \& Molendi 2004). A direct observational link with our proposal that HBIdriven turbulence is present in cluster cores is via turbulent diffusion of metals throughout the core of the cluster. Rebusco et al. (2005) estimate the iron injection rate into the Perseus cluster as a function of radius, and find that significant diffusion of the iron is required to explain the measured radial profile of the iron abundance. They estimate a diffusion constant within the core of $\mathcal{D} \sim 2 \times 10^{29} \mathrm{~cm}^{2} \mathrm{~s}^{-1}$ and show that this can be reasonably accommodated by a turbulent transport picture. The source of turbulence is generally attributed to the central AGN, either by direct stirring of the ICM by AGN jets, or by AGN cosmic-ray driven convection (Chandran \& Rasera 2007). Cluster-cluster mergers have also been considered as a source of turbulence (Fujita, Matsumoto \& Wada 2004).

A distinguishing feature of our model is that it makes a direct connection between the temperature gradient found in cluster cooling cores and turbulent diffusion. The inferred Rebusco et al. (2005) diffusion constant is readily accommodated by eq. (13) if $\xi$ is order $0.1 r$. Moreover, we also predict efficient turbulent transport of metals within the cooling core, but significantly reduced metal transport in the more isothermal regions of the cluster. Thus, the fact that the high metallicity cores appear to be limited to the region where a temperature gradient is maintained (e.g., Baldi et al. 2007; Snowden et al. 2008) is neatly accounted for.

This scenario would require an associated convective heat flux larger than is seen in numerical simulations of noncooling HBI systems (Parrish \& Quataert 2008). Estimates of the ratio of the convective to local conductive fluxes based on equation (12) and the above $\mathcal{D}$ value are 
of order $10^{-2}$ to $10^{-1}$ for cluster cores. The noncooling HBI simulations, by contrast, yield values of order $10^{-3}$. This will be an important point to reconcile in numerical simulations of cooling HBI flows.

Thus far, we have focused exclusively on hot clusters where unbridled Spitzer conductivity is a more than sufficient heat source for the cores. How does our picture relate to clusters more generally? Best et al. (2007) have studied a large sample of clusters from the Sloan Digital Sky Survey (SDSS) and gauged the role of radio-mode feedback in these systems under the very reasonable assumption that the relationship between radio-luminosity and mechanical power is not a strong function of environment. They find that, while embedded radio galaxies can more than offset radiative cooling in galaxy groups and small clusters, the AGN feedback hypothesis has significant energy problems when one considers large/rich clusters. They further show that (when parameterized in terms of the virial velocity of the cluster) the AGN feedback hypothesis becomes untenable just about where the clusters are hot enough for thermal conduction to play a significant role. This coincidence is striking, and strong circumstantial evidence that thermal conduction plays an important role in regulating these hot clusters.

\section{SUMMARY}

Cooling flows in galaxy clusters show remarkably regular temperature profiles. Naive cooling models, in which radiative losses are replenished by advected heat inflow (i.e., classical cooling flow models), fail badly, predicting an excessively large X-ray surface brightness and large amounts of cold gas (or powerful star formation). Direct heating from a central AGN is in principle a potent energy source, but why this should result in regular temperature profiles and temperature floors that scale with the virial temperature, is unclear.

Temperature gradients in the hot gas would produce a large inward heat flux if Coulomb collisions alone were responsible for heat transport. This process, which has in fact been extensively studied, does not currently enjoy widespread support as an important generic heating mechanism. Heating by thermal conduction, when im- portant, seems to be overwhelming, and even if the match is good, thermal stability and fine-tuning remains an issue. For these reasons, researchers have often looked to external sources of heat (e.g., the AGN) as the resolution of the cooling flow problem.

We argue that the recent finding of Q08, that inward directing heat flow in a dilute plasma is unstable and strongly self-regulating, shows great promise as a mechanism to maintain regular temperature profiles. In this work, we have proposed that the combination of a reverse convective heat flux and field-line insulation maintains critical stability conditions corresponding to nearly static stratified atmospheres. In essence, cluster cores are in a state of marginal stability, not to the HBI, but to the radiative HBI. Departures from this state should be stable: an increased diffusive flux would produce a stronger reverse convective flux, and vice-versa. A fully insulated cooling core would draw field lines inward, opening a thermal path. As a by-product of convection, we expect efficient diffusion of metals in regions where a strong temperature gradient exists. This agrees with observations of enhanced ICM metallicities over precisely the same region where the temperature gradient is maintained, but the associated convective heat flux would be larger than those inferred from nonradiative numerical simulations. Radiative HBI simulations are clearly needed.

The basic scenario outlined in this paper- that cluster cores are in a state of marginal stability regulated by the radiative HBI - can be tested numerically by direct simulation. In a subsequent paper, we will report the results of such a study.

\section{ACKNOWLEDGEMENTS.}

This work was supported by a Chaire d'Excellence award to SAB from the French Ministry of Higher Education. CSR thanks the Physics Department of the École Normale Supérieure de Paris for its hospitality and support of a one month visit in March 2008 during which this work was conducted. CSR also acknowledges support from the Chandra Theory and Modeling Program under grant TM7-8009X.

\section{REFERENCES}

Balbus S.A., 2000, ApJ, 534, 420

Baldi A, Ettori S., Mazzotta P., Tozzi P., Borgani S., 2007, ApJ, 666,835

Benson A., et al., 2003, ApJ, 599, 38

Best P.N., von der Linden A., Kauffmann G., Heckman T.M., Kaiser C.R., 2007, MNRAS, 379, 894

Binney J., Cowie L.L., 1981, ApJ, 247, 464

Birzan L., Rafferty D.A., McNamara B.R., Wise M.W., Nulsen P.E.J., 2004, ApJ, 607, 800

Chandran B.D.G., Rasera Y., 2007, 671, 1413

Chuzarov E., et al., 2001

Conroy C., Ostriker J.P., 2008, ApJ in press (arXive:0712.0824)

Fabian A.C., 1994, ARA\&A, 32, 277

Fabian A.C. et al., 2000, MNRAS, 318, L65

Fabian A.C. et al., 2003, MNRAS, 344, L43

Fabian A.C., Sanders J.S., Taylor G.B., Allen S.W., Crawford

C.S., Johnstone R.M., Iwasawa K., 2006, 366, 417

Felten J.E., Gould R.J., Stein W.A., Woolf N.J., 1966, ApJ, 146, 955

Fujita Y., Matsumoto T., Wada K., 2004, ApJ, 612, L9

Gastaldello F., Molendi S., 2004, ApJ, 600, 670

Heinz S., Choi Y.Y., Reynolds C.S., Begelman M.C., 2001, ApJ, 569, L79
Kim W.T., Narayan R., 2003, ApJ, 596, 889

Narayan R., Medvedev M.V., 2001, ApJ, 562, L129

Parrish I.J., Quataert E., 2008, ApJL, submitted (arXive:0712.3048)

Parrish I.J., Stone J.M., 2005, ApJ, 633, 334

Parrish I.J., Stone J.M., 2007, ApJ, 664, 135

Peterson J.R. et al., 2001, A\&A, 365, L104

Peterson J.R., Fabian A.C., 2006, Phys. Rep., 427, 1

Quataert E., 2008, ApJ, 673, 758

Rebusco P., Churazov E., Bohringer H., Forman W., 2005, MNRAS, 359, 1041

Schuecker P., Finoguenov A., Miniati F., Bohringer H., Briel U.G., 2004, A\&A, 426, 387

Snowden S., Mushotzky R.F., Kuntz K.D., Davis D.S., 2008, A\&A, 478, 615

Spitzer L., 1962, Physics of Fully Ionized Gases, 2nd edn., Wiley-Interscience, New York

Tamura T. et al., 2001, A\&A, 365, L87

Vernaleo J.C., Reynolds C.S., 2006, ApJ, 645, 83

Voigt L., Fabian A.C., 2004, MNRAS, 347, 1130

Vogt C., Ensslin T.A., 2005, A\&A, 434, 67

Young A.J., Wilson A.S., Mundell C.G., 2002, ApJ, 579, 560

Zakamska N.L., Narayan R., 2003, ApJ, 582, 162 